\title{
Top-down and bottom-up modulation in processing bimodal face/voice stimuli
}

Marianne Latinus ${ }^{1,2^{*}}$, Rufin VanRullen ${ }^{1}$, Margot J Taylor $^{3}$

\begin{abstract}
Background: Processing of multimodal information is a critical capacity of the human brain, with classic studies showing bimodal stimulation either facilitating or interfering in perceptual processing. Comparing activity to congruent and incongruent bimodal stimuli can reveal sensory dominance in particular cognitive tasks.

Results: We investigated audiovisual interactions driven by stimulus properties (bottom-up influences) or by task (top-down influences) on congruent and incongruent simultaneously presented faces and voices while ERPs were recorded. Subjects performed gender categorisation, directing attention either to faces or to voices and also judged whether the face/voice stimuli were congruent in terms of gender. Behaviourally, the unattended modality affected processing in the attended modality: the disruption was greater for attended voices. ERPs revealed topdown modulations of early brain processing (30-100 ms) over unisensory cortices. No effects were found on N170 or VPP, but from 180-230 ms larger right frontal activity was seen for incongruent than congruent stimuli.

Conclusions: Our data demonstrates that in a gender categorisation task the processing of faces dominate over the processing of voices. Brain activity showed different modulation by top-down and bottom-up information. Top-down influences modulated early brain activity whereas bottom-up interactions occurred relatively late.
\end{abstract}

\section{Background}

The ability to integrate information from several sensory modalities is a vital skill of the human brain, as information we receive from the external world is often multimodal. Although there has been a recent surge of research focusing on the processing of multimodal information, our knowledge of the neural substrates underlying this ability for complex stimuli in humans is still limited.

Researchers have used two main paradigms to investigate multimodal processing. One is designed to assess the perceptual gain of multisensory inputs by comparing the behaviour and the neural activity evoked by multimodal and unimodal inputs $[1,2]$. The other paradigm assesses the competition between senses using bimodal stimuli which could be either congruent or incongruent; using incongruent stimuli can reveal the existence of a cross-modal bias [3]. These two approaches yield different information: the first determines the advantages and limits of multimodality, while the second provides

\footnotetext{
* Correspondence: marianne@psy.gla.ac.uk

'Université de Toulouse, UPS, CNRS, Centre de recherche Cerveau et Cognition, Toulouse, France
} Cognition, Toulouse, France

information on sensory dominance and its influence on task performance. The present study investigates sensory competition or dominance in the processing of gender in bimodal face/voice stimuli.

Sensory dominance has been largely studied in terms of spatial localisation or temporal discrimination. The research approach of comparing congruent and incongruent bimodal stimuli has demonstrated that the influence of the senses is asymmetric and task-dependent. For example, in ventriloquism, the visual-spatial information biases the localisation of the source of auditory information toward the source of visual information [4-6]. The localisation of a visual stimulus is however, almost unaffected by simultaneous discordant auditory information [4]. In contrast, in the temporal domain, the auditory modality dominates the visual, i.e. when subjects judge temporal aspects of a stimulus (frequency of occurrence, temporal frequency, etc.), auditory stimuli modulate perceived information in the visual modality [7-9]. These results suggest that in the spatial domain, vision dominates audition, while in the temporal domain, the reverse is true [10]. Using emotional faces and voices, it has been demonstrated that a static face 
alters the perception of vocal emotion even when the task required ignoring the face $[3,11]$. One aim of the present study was to determine if sensory dominance could be observed in the processing of faces and voices, i.e. is the influence of one sensory modality on the other equivalent or symmetrical in the perception of gender? To this purpose, we manipulated attention through task demands on congruent and incongruent face/voice stimuli.

Neural correlates of multimodal processing have been investigated using fMRI, PET and ERPs, with results showing that bimodal processing was task-sensitive [12]. As in the behavioural literature, various approaches have been used to study neural mechanisms underlying multimodal processing. Comparing the brain activity for bimodal stimuli to the sum of activity for unimodal stimuli (e.g., AV - $(\mathrm{A}+\mathrm{V})$ ) revealed that congruent bimodal stimuli enhanced brain activity either in sensory-specific cortices $[1,13,14]$ or in brain regions described as heteromodal [15]. The timing of this bimodal activation was very rapid, affecting brain processing within $40 \mathrm{~ms}$ [1,16-18]. Even with more biological stimuli (sounds and pictures of animals), early interactions between visual and auditory processing were seen on the visual N1 component ( 150 ms) [19].

Investigations of higher-level multimodal processing critical to human social interactions (faces and voices) have been less common, with most studies on face and voice integration focussed on speech processing. The interaction between visual and auditory stimuli in the speech domain is classically demonstrated by the McGurk effect [20]. As seen with simple bimodal object and spatial processing, there is a behavioural advantage of bimodal redundant speech [21]. Audiovisual integration of faces and voices has also been shown in nonhuman primates, as monkeys are able to match a face and a certain vocalisation [22], demonstrating its wider application to other social species. The small literature on face/voice interactions in a non-verbal context is largely focussed on emotional processing [23-25]. Emotion expression protocols have also been used with monkeys, as Parr (2004) showed, in a match-to-sample task, a modality preference depending on the expression to be matched [26]. Bidirectional interference in processing has been demonstrated with incongruent emotional voices and faces [27] suggesting no sensory dominance in the processing of emotions. Congruent emotional faces and voices enhance the auditory N1 [11,25]; yet, in a bimodal speech perception study, the opposite was demonstrated, a reduced N1 to congruent bimodal stimuli [21].

Although face/voice associations to extract non-speech information have been rarely studied, there is a wealth of face and voice processing studies in unimodal paradigms. A large literature provides evidence that faces are processed through a distributed and hierarchical network [28]; neurophysiological studies provide latencies for the different stages of face processing. The N170 component is sensitive to a range of manipulations of faces [29-32] suggesting that it reflects automatic face processing [33,34]. Earlier components have also been reported to be face-sensitive $[35,36]$.

Comparable studies have been completed with voices, often referred to as 'auditory faces' due to the similarity of information carried by faces and voices $[37,38]$, and have revealed that the processing of non-speech information of voices involved structures located along the right superior temporal sulcus. There are few ERP studies comparing voices to other auditory stimuli. Two papers report a positive deflection $320 \mathrm{~ms}$ after stimulus onset that is larger to voices than to musical instrument stimuli, labelled the Voice Selective Response (VSR) $[39,40]$. A recent study comparing voices to various non-vocal sounds suggests that the voice/non-voice discrimination could occur earlier, in the latency range of the auditory P2, 160-240 ms [41]. The processing of faces and voices seems thus to draw on specialised and distinct brain regions and to have distinct temporal profiles.

The integration of information from faces and voices is a crucial skill that is essential for normal social interactions. Determining how cross-modal processing of faces and voices occurs will contribute significantly to our understanding of this critical human ability. Here we investigated the effects of attention on the perception of bimodal congruent and incongruent face/voice stimuli (see Figure 1) using three gender judgement tasks. Gender discrimination is a common task in unimodal studies, as it requires some depth of processing, but is readily done. In the first task, subjects judged if the gender of the face and the voice were congruent or not. In the second and third tasks subjects categorised the bimodal stimuli by gender, in one case attending only to voices or, conversely, attending only to the faces. The same stimuli were used in the three tasks allowing us to determine effects due only to the task, i.e. topdown influence on the processing of bimodal stimuli. The directed attention aspects of the tasks allowed us to determine the influence of top-down modulation on multimodal processing, whereas the use of congruent and incongruent stimuli provided information on bottom-up stimulus-dependent processing. The use of only congruent and incongruent bimodal stimuli does not allow a direct comparison of responses to bimodal versus unimodal stimuli.

We hypothesized that if vision dominates over audition in gender perception, an incongruent face would disrupt the processing of voice gender while an 


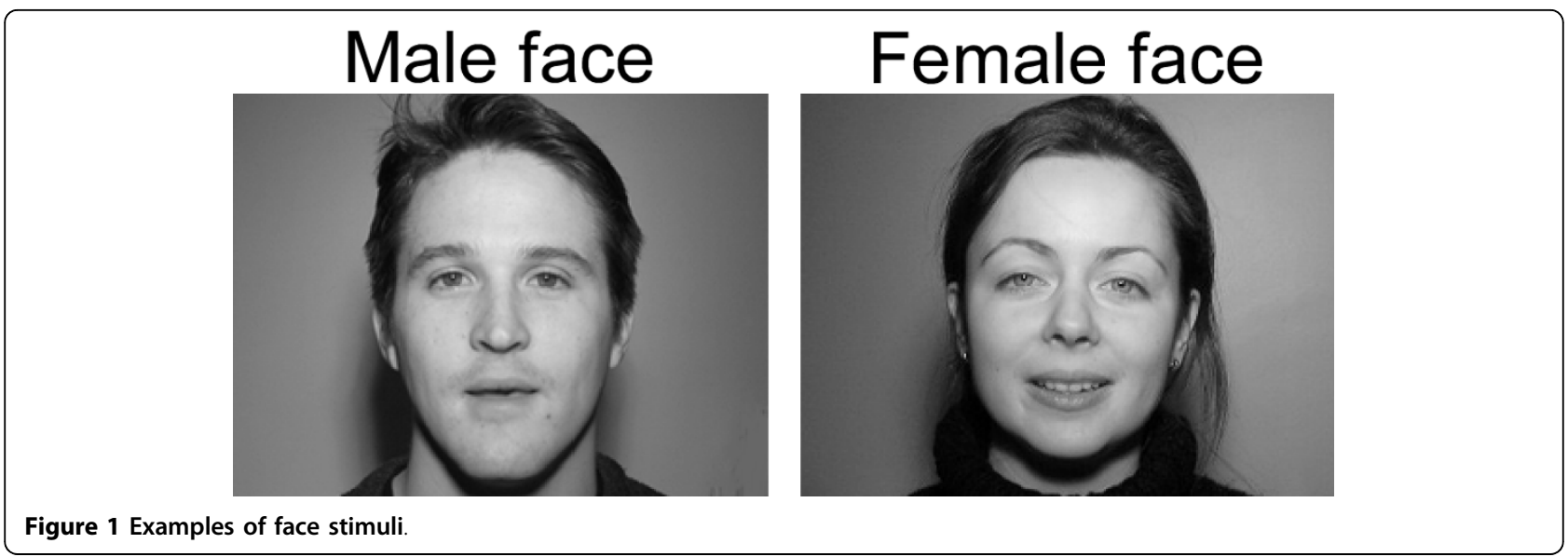

incongruent voice would have less impact on the perception of face gender. On the other hand, if incongruence has a similar effect regardless of whether subjects performed the task on faces or voices, this would suggest an equivalent influence of the two senses on each other. We also hypothesized that directing attention to one or the other modality would modulate brain activity earlier than stimulus congruency. We showed that directing attention to only one modality modulated early ERPs that were more representative of the attended modality. The congruency task required the processing of both auditory and visual information and the pattern of cerebral activity reflected interaction effects. Comparing congruent and incongruent stimuli allowed us to show that faces dominate over voices in the integration of auditory and visual information of gender, and also demonstrated that bottom-up or automatic processing of the bimodal stimuli arose later $(\sim 180 \mathrm{~ms})$ in right frontal regions.

\section{Results}

\section{Behavioural results}

Subjects were equally accurate with gender categorisation of faces (96.47\%) and of voices (95.44\%); congruency judgement in the $\mathrm{BOTH}$ condition was more difficult, reflected by the lower percentage of correct responses $\left((90.05 \%) \mathrm{F}_{2,36}=15.96, \mathrm{p}<0.001-\right.$ Table 1$)$. Congruency of the face and voice affected gender categorisation performance only during the VOICE task (attention $\times$ congruency: $\left.\mathrm{F}_{2,36}=7.92, \mathrm{p}=0.002\right)$ : incongruent face information impaired gender categorisation of voices (congruent: 97.49\%; incongruent: $93.38 \%$, difference $=4.11 \%, 95 \% \mathrm{CI}$ of the difference $=$ [1.52 7.12]) - see Figure 2a, and Table 1. This impact of incongruent information on subjects' accuracy in the VOICE and not in the FACE task, demonstrated an asymmetry in the processing of faces and voices.

Reaction times (RTs) were influenced by task $\left(\mathrm{F}_{2,36}=\right.$ 63.09, $\mathrm{p}<0.001$ ), being longer in the BOTH task, as the congruency judgment took longer than gender categorisation (paired comparisons, $\mathrm{p}<.05$ - Table 1). Gender categorisation took longer for voices than faces (difference $=151.15 \mathrm{~ms}, 95 \% \mathrm{CI}=[112.71191 .11], \mathrm{p}<.0001-$ Figure 2b, Table 1). Finally, incongruent stimuli took longer to categorise for all three tasks regardless of attentional conditions $\left(\mathrm{F}_{1,18}=35.89, \mathrm{p}<0.001\right.$ - difference $=44.89 \mathrm{~ms}, 95 \% \mathrm{CI}=\left[\begin{array}{ll}30.46 & 59.28\end{array}\right]$ ); thus, the bimodal information was processed regardless of whether it was required for the task performance, suggesting an automaticity in face and voice processing.

\section{Neurophysiological results}

Across the three tasks the waveform morphology was similar to that observed in face ERP studies; P1, N170, P2 components recorded from posterior electrodes and N1, VPP, N2 from central electrodes (Figure 3). Spatiotemporal analyses revealed differences in brain activity starting as early as $30 \mathrm{~ms}$ after stimulus onset. Task modulated brain activity between 30 and $100 \mathrm{~ms}$ and between 160 and $250 \mathrm{~ms}$. Stimuli, i.e. congruency

Table 1 Hits and Reaction Times for each attentional task and congruence

\begin{tabular}{|c|c|c|c|c|c|c|}
\hline & \multicolumn{2}{|c|}{ To Voices } & \multicolumn{2}{|c|}{ To Faces } & \multicolumn{2}{|c|}{ To Both } \\
\hline & Congruent & Incongruent & Congruent & Incongruent & Congruent & Incongruent \\
\hline Hits (\%) & $\begin{array}{c}97.49 \\
\pm 0.69\end{array}$ & $\begin{array}{r}93.38 \\
\pm 1.65\end{array}$ & $\begin{array}{c}97.12 \\
\pm 0.67\end{array}$ & $\begin{array}{c}95.84 \\
\pm 0.84\end{array}$ & $\begin{array}{c}89.41 \\
\pm 1.33\end{array}$ & $\begin{array}{c}90.68 \\
\pm 1.42\end{array}$ \\
\hline RTs (ms) & $\begin{array}{r}745.68 \\
\pm 33.9\end{array}$ & $\begin{array}{c}790.13 \\
\pm 30\end{array}$ & $\begin{array}{r}594.63 \\
\pm 23.4\end{array}$ & $\begin{array}{c}638.87 \\
\pm 28.07\end{array}$ & $\begin{array}{c}863.31 \\
\pm 35.54\end{array}$ & $\begin{array}{c}909.29 \\
\pm 31.22\end{array}$ \\
\hline
\end{tabular}




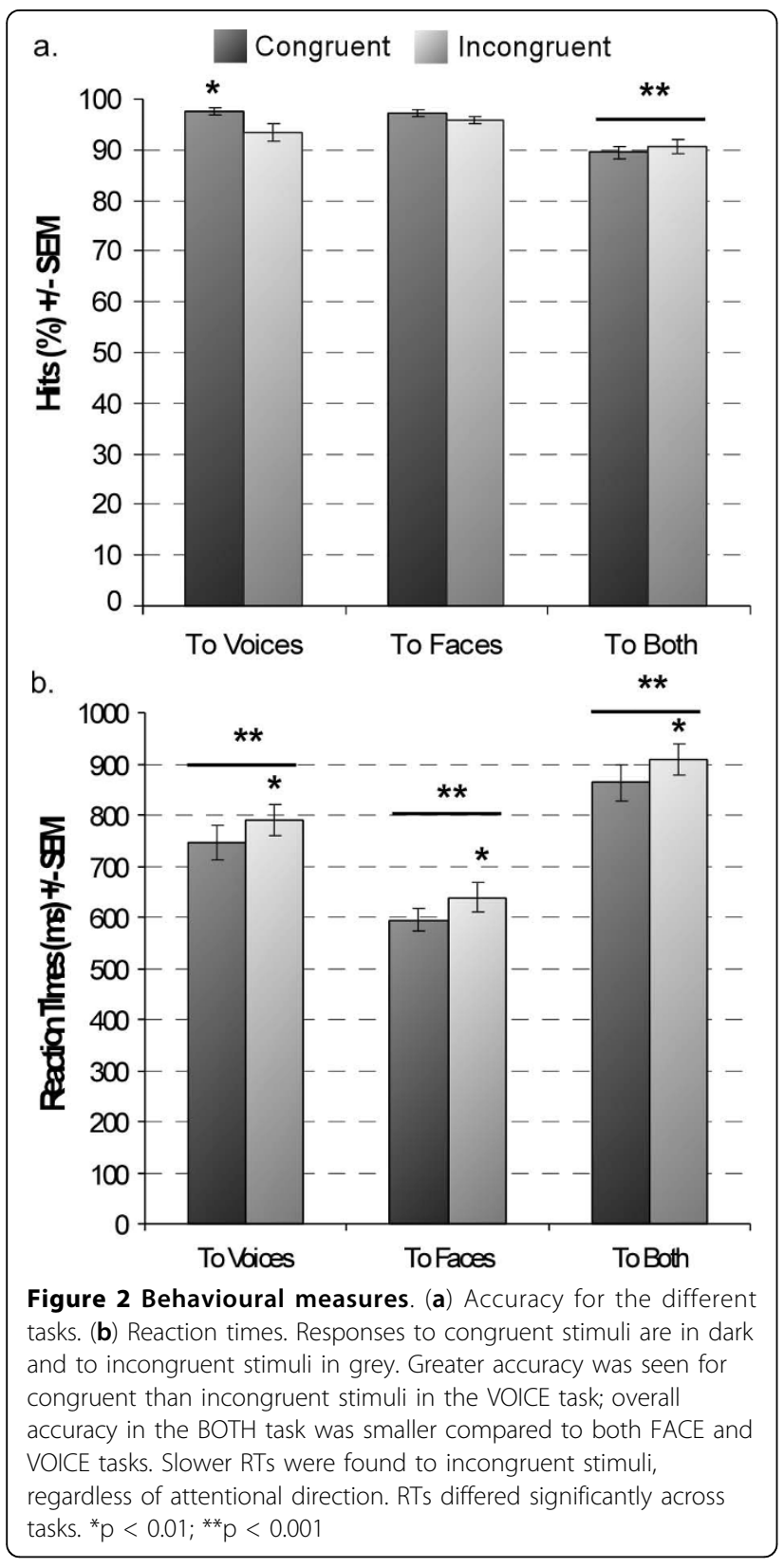

between face and voice, affected brain activity mostly between 150 and $210 \mathrm{~ms}$ (see Figure 4b). Both spatiotemporal and peak analyses showed a modulation of brain activity by task and/or stimuli at a number of locations and latency ranges, as detailed below.

\section{Early effects, P1 and N1 components}

$\mathrm{P} 1$ amplitude varied with attention as it was larger in the FACE and BOTH tasks than in the VOICE task $\left(\mathrm{F}_{36,2}=8.37, \mathrm{p}=0.001\right)$ - Figure $3 \mathrm{a}$. The auditory $\mathrm{N} 1$ was larger in the FACE task than in the BOTH and VOICE tasks $\left(\mathrm{F}_{36,2}=4.075, \mathrm{p}=0.029\right.$ - Figure 3b). $\mathrm{P} 1$ was largest at $\mathrm{PO} 7 / \mathrm{PO} 8$ regardless of where attention was directed; however, in the FACE and BOTH conditions, the $\mathrm{P} 1$ was second largest at $\mathrm{O} 1 / \mathrm{O} 2$, whereas for the VOICE condition $\mathrm{P} 1$ at $\mathrm{PO} / \mathrm{PO} 8$ and $\mathrm{PO} 3 / \mathrm{PO} 4$ were equivalent and larger than at $\mathrm{O} 1 / \mathrm{O} 2$ (attention $\times$ electrodes: $\mathrm{F}_{72,4}=5.25, \mathrm{p}=0.006$ ) (see Table 2). In other words, P1 was largest occipitally in conditions with attention directed to faces. The more anterior topography when subjects attended to voices may reflect overlapping activation of auditory brain areas for early auditory processing. Congruency affected neither P1 $\left(\mathrm{F}_{18,1}=2.357\right.$, n.s. $)$ nor $\mathrm{N} 1\left(\mathrm{~F}_{18,1}=0.378\right.$, n.s. $)$ amplitude. Neither P1 nor N1 latencies were affected by attention or congruency (Figure 3).

In the spatio-temporal analyses, early differences were observed over central and posterior temporal brain areas (Figure 4b, 5a) between $30 \mathrm{~ms}$ and $90 \mathrm{~ms}$. Post-hoc analyses revealed that the topography differed mostly between the FACE and the VOICE condition (Figure $5 b)$. In Figure 5c, it is evident that the topography for FACE and VOICE are quite distinct and representative of the topography observed for unimodal visual and auditory stimuli at this latency. The topography in the BOTH condition approaches the average of FACE and VOICE topographies in the same latency range (see Figure $5 \mathrm{c}$, far right map); a difference between the BOTH and FACE tasks can be observed over fronto-central regions (Figure $5 \mathrm{c}$, centre two maps).

\section{N170/VPP}

N170 was earlier when attention was directed towards both faces and voices (BOTH - $147.6 \mathrm{~ms}$ ) than when it was directed towards faces (FACE - $150.7 \mathrm{~ms}$ ) or voices $($ VOICE - $155.1 \mathrm{~ms})$ alone $\left(\mathrm{F}_{36,2}=6.93, \mathrm{p}=0.006\right)$ (Fig. 6a). N170 latency was shorter in the right hemisphere $\left(\mathrm{RH}-149.9 \mathrm{~ms}, \mathrm{LH}-152.4 \mathrm{~ms} ; \mathrm{F}_{18,1}=5.25\right.$, $\mathrm{p}=0.034$ ) (Figure 3a). VPP peaked earlier when attention was directed to faces $(154.9 \mathrm{~ms})$ and to both faces and voices $(154 \mathrm{~ms})$, relative to when attention was directed only towards voices $(160 \mathrm{~ms})\left(\mathrm{F}_{36,2}=4.45, \mathrm{p}=0.04\right)$ (Figure $6 \mathrm{~b})$. N170 and VPP amplitudes were not significantly affected by task or stimulus (Figure 6a and 6b).

\section{Later effects; visual and auditory P2s, VSR}

Neither attention nor congruency affected the visual P2 or the VSR significantly. Both components showed hemisphere effects, however. The visual P2 was larger in the right than in the left hemisphere $\left(\mathrm{F}_{1,18}=8.54, \mathrm{p}=0.009\right)$; the VSR had a shorter latency $\left(\mathrm{F}_{1,18}=10.4, \mathrm{p}=0.005\right)$ and larger amplitude $\left(\mathrm{F}_{1,18}=17.42, \mathrm{p}=0.001\right)$ over the right hemisphere. The auditory $\mathrm{P} 2$ has been proposed to index voice processing [42], yet it was not apparent in our study. We reasoned that the auditory P2 may be masked by the VPP, which occurs in a similar latency range and over the same electrodes. 


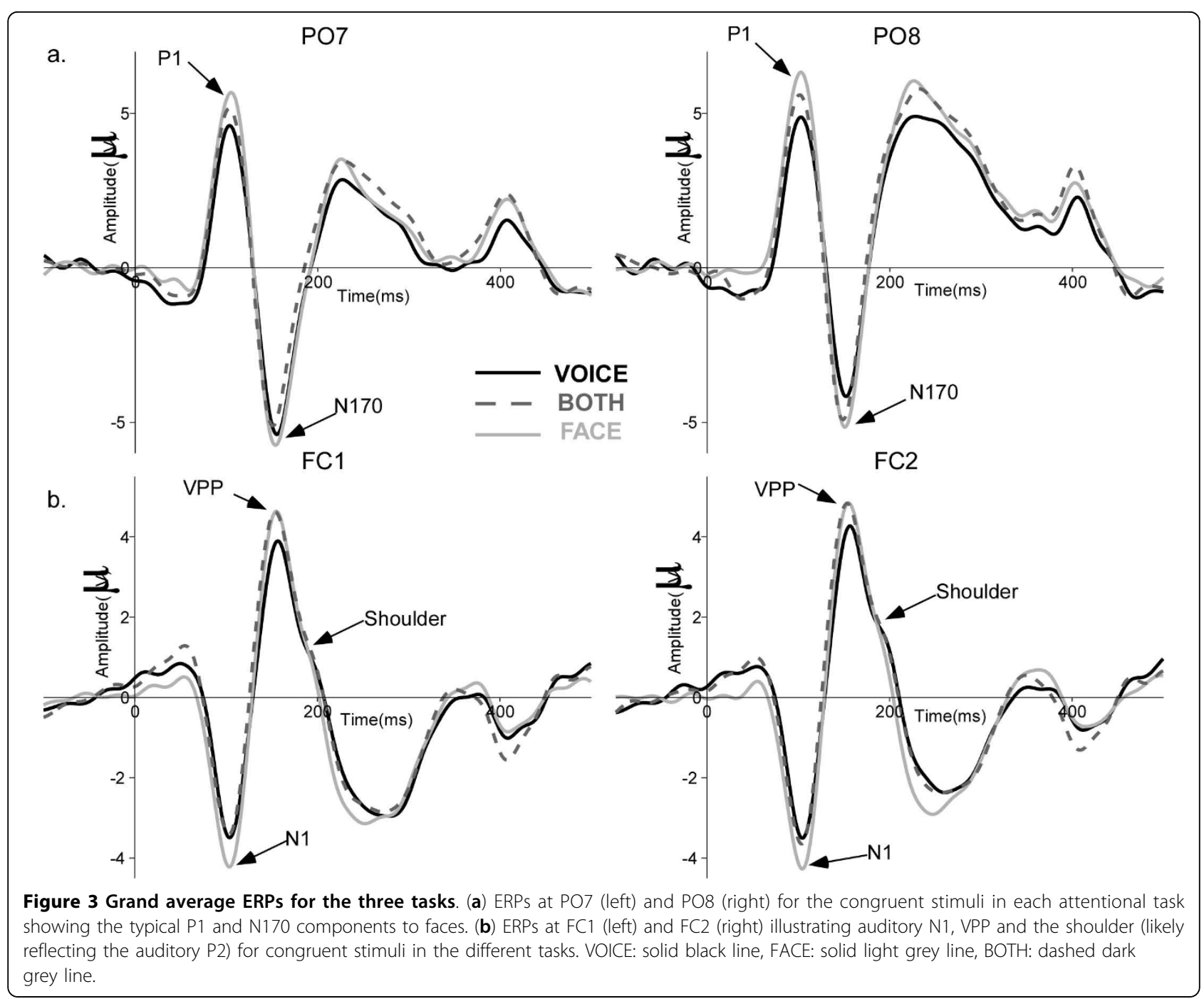

Spatio-temporal analyses of ERP topography between 170 and $220 \mathrm{~ms}$ showed a larger negativity in the BOTH task compared to FACE and VOICE tasks at frontal electrodes (Figure 6c). Post-hoc tests revealed significant differences between VOICE and BOTH on bilateral posterior electrodes as well as differences between VOICE and FACE on bilateral temporal electrodes (Figure 6c). A stimulus-driven congruency effect showed a significantly increased positivity to incongruent stimuli in the P2 latency range between 182 and 230 $\mathrm{ms}$, in right centro-temporal areas associated with an increased negativity in left posterior regions (Figure 6d).

\section{Discussion}

This study investigated the influence of top-down and bottom-up processes on the important human ability of integrating multimodal face/voice stimuli. Top-down influences were manipulated by the task requirements; stimuli were the same in all three tasks, only attentional instructions differed. Bottom-up influences were evident in the processing of congruent versus incongruent stimuli, i.e. how stimulus characteristics influenced the interaction between modalities.

\section{Top-down and bottom-up influences on behaviour}

Behavioural data showed that directing attention toward the auditory or visual modality biased the processing of the bimodal face/voice stimuli. With the same bimodal stimuli in the tasks, we showed that RTs were shorter when attention was directed to faces than voices (regardless of congruency). This is in accordance with other reports studying bimodal natural object recognition $[19,27,43]$ showing that visually based categorisation is faster than auditory based categorisation. RTs were longer for incongruent stimuli regardless of the direction of attention; thus, the unattended modality affected processing in the attended modality, revealing the automatic processing of bimodal information [27]. 


\section{a. Electrode locations}

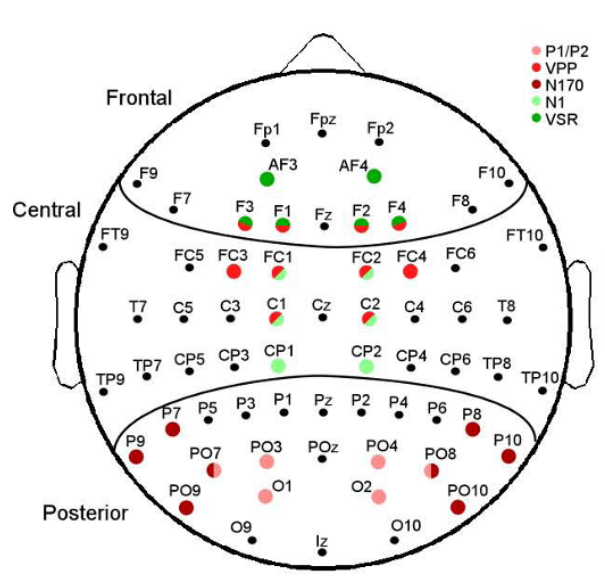

\section{b. Results of the bootstrapped 2-way ANOVA}

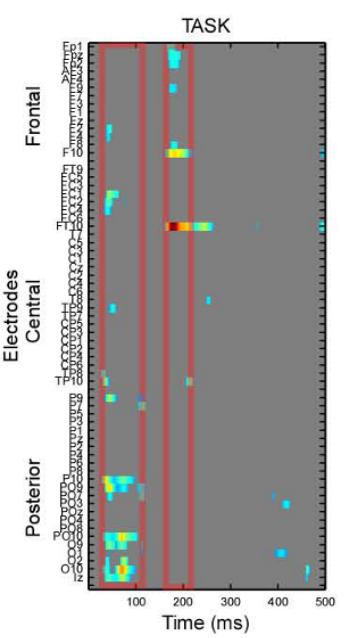

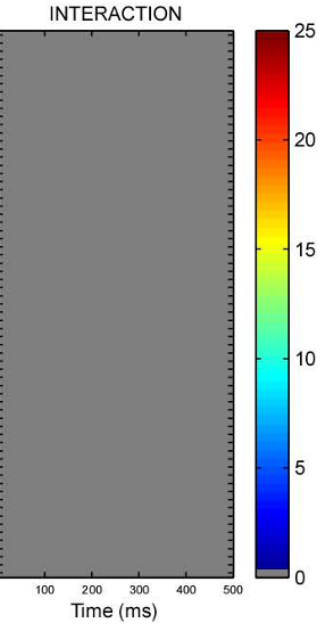

Figure 4 Results of the bootstrapped ANOVA for the 2 factors and their interaction. (a) Electrode locations. Red: electrodes on which visual components were measured. Green: electrodes on which auditory components were measured. (b) Results of the bootstrapped 2-way ANOVA. The scale represents F-values, when the 2-way ANOVA was significant after correction for repeated measures, for factor task and stimulus as well as the interaction. Non-significant F-values are presented in grey. Red rectangles indicate latencies of interest, determined by more consistent (spread over several electrodes and time points) and larger effects. This shows both early (30-90 ms) and later (170-220 ms) task effects, stimulus effects at 180-230 ms and no interaction.

Incongruent information modulated subjects' accuracy according to the task. Accuracy was lower in the VOICE task when the voice was presented with an incongruent face, an effect not seen in the FACE task.

This result suggests asymmetrical interference between the processing of faces and voices in gender recognition: faces impact the processing of voice gender more than the reverse. A recent study using ambiguous faces showed that low-level auditory features influence the perception of face gender [44]. Although this result could be seen as opposite to ours, this is not the case as the gender of the faces in the Smith et al. study was ambiguous and thus, gender attribution was mostly based on auditory cues. Asymmetrical interference effects have been reported in studies using various paradigms and stimuli, and have been understood as reflecting a sensory dominance in the processing of particular features $[8,18,43]$. Our results demonstrated that in gender categorisation of faces and voices, visual information dominates auditory information. This dominance of faces over voices for gender discrimination could be explained by different hypotheses of sensory dominance.

Table 2 P1 amplitude as a function of electrode in the different attentional tasks

\begin{tabular}{cccc}
\hline Electrodes & To Voices $(\boldsymbol{\mu} \mathbf{V})$ & To Faces $(\boldsymbol{\mu V})$ & To Both $(\boldsymbol{\mu V})$ \\
\hline O1/O2 & $4.827 \pm .643$ & $6.018 \pm .754$ & $5.431 \pm .808$ \\
\hline PO3/PO4 & $5.223 \pm .614$ & $5.667 \pm .697$ & $4.992 \pm .720$ \\
\hline P07/PO8 & $5.349 \pm .642$ & $6.999 \pm .559$ & $6.251 \pm .723$ \\
\hline
\end{tabular}

One is the information reliability hypothesis, which suggests that the dominant modality is whichever is more appropriate and the more efficient for the realisation of the task [45]. In our study the more reliable modality would be vision due to intrinsic properties of the stimuli; information required to perform gender categorisation are easily and immediately extracted from a face, whereas auditory stimuli are always dynamic and thus some number of cycles need to be heard before a voice could be recognised by gender. Another possible hypothesis for the visual dominance would be that sensory dominance results from top-down influences [45]. However, if a stimulus automatically captures attention in one modality (such as faces in the present case), the processing of that stimulus would occur despite attention instructions, and any dominance due to attention would be reduced. This latter explanation is in accordance with studies demonstrating that gender categorisation of faces occurs in the near absence of attention; that gender is automatically extracted from faces [46]. Thus, the automatic processing of faces [47] would reduce or mask the processing in the auditory modality even when attention was explicitly directed to the voices.

The hardest of the three tasks was to determine if the gender of both face and voice was congruent, reflected by this task's lower accuracy and longer RTs. In other multimodal studies, a behavioural facilitation is often reported with bimodal stimuli $[1,18,48]$. However, in tasks involving identification of a non-redundant target, 

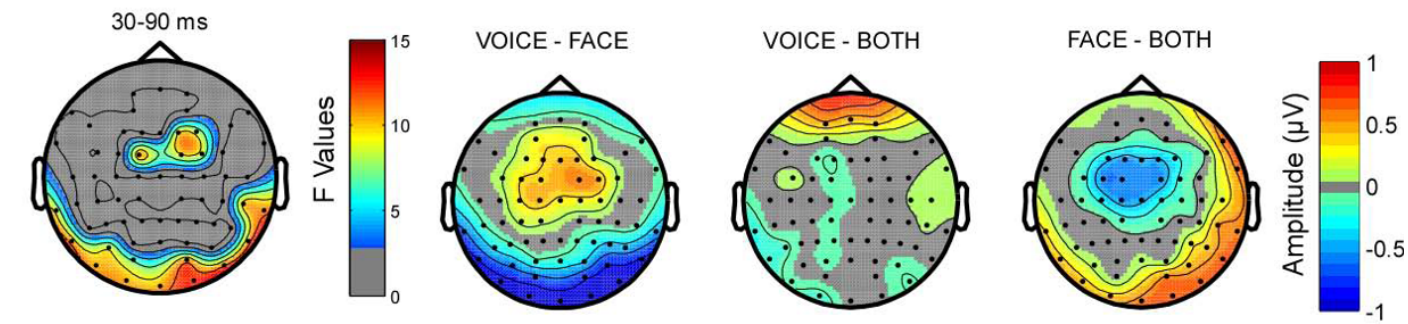

c. Average topography for each task
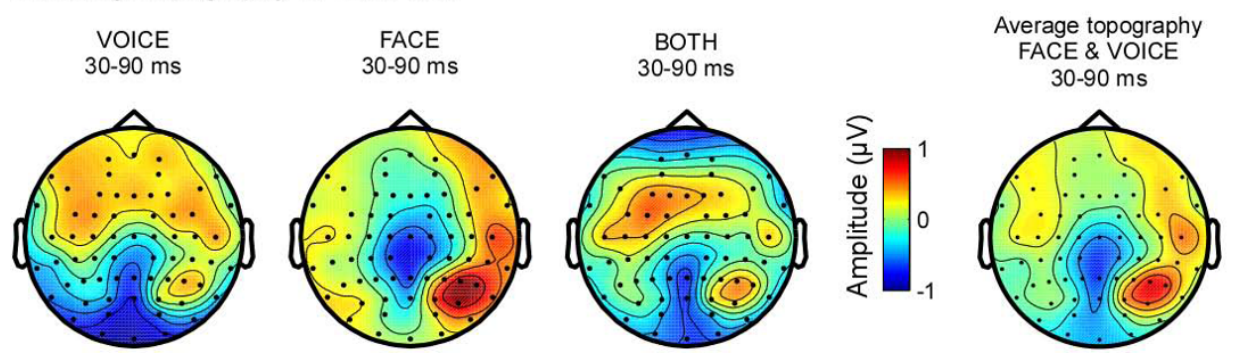

Figure 5 Attention modulated early brain activity $(\mathbf{3 0 - 9 0} \mathbf{~ m s}$ ). (a) Topography of the average F-values in this time range. Non-significant F-values are in grey. (b) Topography of the absolute differences between the two tasks where the p-values of the post-hoc test were significant $(p<0.05)$. Non-significant data are represented in grey. (c) Average topographic maps for each task between 30 and 90 ms. Left to right: FACE, VOICE, BOTH and the average between FACE and VOICE, shown as a comparison. Over posterior regions, the map for the BOTH task is similar to the map for the FACE task, while in fronto-central regions it is more similar to the map for VOICE. Comparison of BOTH with the average of VOICE and FACE shows that the topography in the BOTH task differed from the average topography of the other tasks over fronto-central electrodes.

accuracy is reduced [49] and RTs are generally longer [16]. In the BOTH task, subjects were not identifying a single target but making a congruency judgement which required the extraction of relevant information from both modalities; it is consistent with the literature that this task was the most difficult.

Behavioural results provide evidence of a modulation of the responses by both top-down and bottom-up influences. Bottom-up incongruent information delayed the processing of gender in the attended modality regardless of attention instructions. Top-down processes also impacted gender categorisation of bimodal face/voice stimuli. We suggest that directing attention to a specific sensory modality led to a competition in attentional resources, particularly evident in the VOICE condition. As face processing appears mandatory [50], some attentional resources are automatically allocated to faces, which may account for voice processing being less efficient than face processing with the bimodal stimuli. Directing attention to both auditory and visual modalities (BOTH task) led to longer RTs and lower accuracy, again likely reflecting dispersed attentional resources.

\section{Top-down and bottom-up influences on ERPs}

The ERP waveforms, regardless of the task, were very similar to those described in the face literature $[29,32]$.
This supports the suggestion that in our paradigm face processing dominated over voice processing, in accordance with the conclusions from the behavioural data.

\section{Modulation of brain activity by top-down processes}

Neurophysiological responses were modulated by task as early as $30 \mathrm{~ms}$, as seen in the dissimilar topographies as a function of the direction of attention. Various studies have reported very early activity reflecting bimodal integration when comparing the response to bimodal stimuli to the sum of responses to unimodal stimuli $[1,17,18,51]$. Early multimodal effects were explained either as anticipatory effects [17] or as recruitment of a novel population of neurons by bimodal stimuli in the visual cortex [1]. In the present study, this early modulation reflected top-down processes, as we found early activation of unisensory cortices of the attended modality attributable to preparatory processes. This is in accordance with fMRI and ERP data showing attentionrelated modulations in modality-specific cortices for bimodal stimuli $[49,52,53]$. In the VOICE task, the observed brain topography to the bimodal stimuli showed a larger activity in fronto-central brain regions, whereas in the FACE condition, activity to the bimodal stimuli was larger in right occipital regions. Thus, directed attention to either vision or audition led to greater 


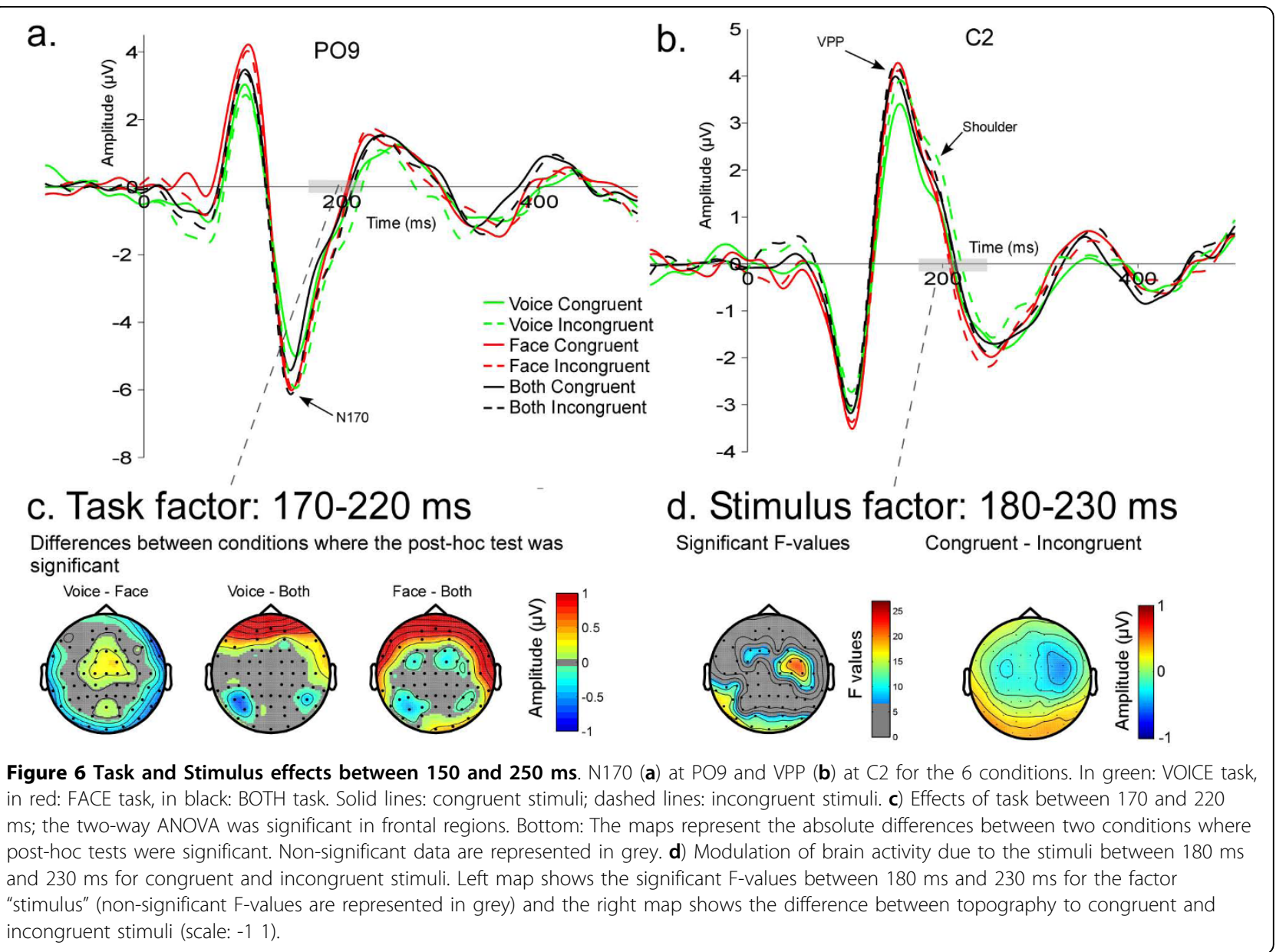

activation in the respective modality-specific cortices; based indirectly on comparing our results with results in the literature, as we did not use unimodal stimuli. Topography in the BOTH task differed slightly from the average topography of FACE and VOICE condition particularly over fronto-central regions, which might reflect greater attention to voices in the congruency judgment task, as processing voices is less automatic than faces. This is in accordance with the conclusion of the behavioural discussion; directing attention to both faces and voices led to a spread of attention, seen neurophysiologically as an intermediate topography observed for the BOTH task. The early effects in the present study demonstrated that subjects are able to direct their attention to a specific modality; brain activity for the different tasks being representative of the unimodal activity. This is an important finding and justifies the use of paradigms involving directed attention to one sensory modality.

The early visual P1 was larger when attention was directed to faces, seen in FACE and BOTH tasks, consistent with ERP studies showing a larger amplitude for attended versus non-attended stimuli [53]; yet the early auditory $\mathrm{N} 1$ amplitude did not show modulation by attention. P1 topography differed across the conditions: P1 in FACE and BOTH was maximal over occipital electrodes whereas P1 in the VOICE task was more parietal. These topographical differences suggested overlapping components affecting the P1 in the VOICE compared to the other two tasks. Furthermore, the three tasks impacted P1 and N1 differently, suggesting a modulation of the N1/P1 complex in central regions by the processing of auditory information. The fronto-central N1 recorded in the present study may be the negative counterpart of the P1, generally observed with visual stimuli [54], or may reflect auditory processing [55]. Unimodal studies of auditory processing find that auditory $\mathrm{N} 1$ is enhanced to attended auditory stimuli [56]. The absence of differences on the $\mathrm{N} 1$ across the conditions may be due to either a deactivation of auditory cortex when attention was directed to faces or a greater activation of auditory cortex when attention was directed to voices; effects which would cancel each other out, leaving no apparent changes to the bimodal stimuli. 
N170 and VPP peaked earlier when attention was directed to both faces and voices (BOTH task), but no amplitude effects were seen. N170 reflects an automatic processing of faces as demonstrated by various studies [57], and its amplitude is not modulated by attention $[34,50]$; thus, we did not expect a difference in N170 across tasks. In contrast, task affected brain activity around $100 \mathrm{~ms}$, in accordance with studies showing that attention modulates the processing of audiovisual stimuli at different latencies [53].

The auditory P2 was not seen in our data; it probably was obscured by the presence of the VPP. However, we observed a shoulder in the descending slope of the VPP around the auditory P2 latency (180/190 ms [58]) that may correspond to processes normally underlying P2 in unimodal conditions, such as voice processing [42]. Visual inspection of the grand average ERPs revealed that the shoulder was larger in VOICE and BOTH conditions than in the FACE condition; a larger shoulder would imply increased voice processing. In accordance with this suggestion, in the FACE task, the shoulder appeared to be more evident for incongruent stimuli, implying that voices were still processed when they carried incongruent information irrelevant for the task, consistent with the longer RTs in the FACE condition for incongruent stimuli.

The processing of paralinguistic information of faces and voices is shown to be dependent on the sensory modality to which the attention is directed. Moreover, our data showed that the interaction between the processing of faces and voices is asymmetrical with greater influences of visual information than of auditory information. The modulation of bimodal integration by topdown influences could reflect a general mechanism underlying multimodal integration; it is the first time that multimodal ERPs are shown to be task-dependent in the processing of faces and voices at a relatively lowlevel of processing.

\section{Modulation of brain activity by bottom-up processes} Congruency affected brain activity between 180 and $230 \mathrm{~ms}$ after stimuli onset: incongruent stimuli evoked a more positive activity than congruent stimuli in right anterior frontal regions. fMRI studies using bimodal stimuli have shown that the processing of incongruent and congruent stimuli differed in activation in the inferior frontal gyrus (IFG) and the anterior insula [13,59-61], areas thought to be heteromodal. Activity in these regions decreased for incongruent stimuli $[15,62]$. The localisation of the modulation of brain activity by congruency in the present study is compatible with the suggestion that differences between congruent and incongruent stimuli arise from insula or right IFG, and provides a latency $(190 \mathrm{~ms})$ to the previously described effect in the fMRI literature. This result is also in accordance with other ERP studies that reported differences due to congruency over frontal regions before $200 \mathrm{~ms}$ [63]. The inferior frontal gyrus and insula in the left hemisphere are thought to reflect the retrieval and manipulation of linguistic semantic representations $[64,65]$. Other studies demonstrated the role of right insula and IFG in the detection of asynchrony between auditory and visual stimuli [66]. Our data suggest that those regions could also be involved in more general mismatch judgment such as congruency judgment in terms of gender.

\section{Limitations}

One limitation of the study is the use of natural stimuli that can introduce physical differences between the conditions (e.g. between male and female faces or voices). We were interested in the perception of gender on bimodal face/voice stimuli under normal, ecological conditions; this study allows us to show that using these more natural, less tightly controlled stimuli a bias was observed toward faces in the perception of gender. This result suggests that in everyday life situations the perception of gender from faces will dominate over voices. Further study should investigate the perception of gender on more controlled stimuli: for example by using normalised faces and voices, or by controlling the timbre of individual voices, in order to make the tasks equally difficult across sensory modalities. We believe that this could be assessed by using faces in which all "cultural" cues of gender have been removed and by using vowels instead of words.

Another limitation is the fact that we used only bimodal stimuli. Because we were interested in sensory dominance we did not include unimodal conditions to directly compare responses to bimodal stimuli to responses to unimodal stimuli. It should be noted, first, that the lack of unimodal conditions does not prevent drawing conclusions on the sensory dominance in the perception of voice gender, and second, that the rich literature on both face $[29,32,67]$ and voice perception $[41,68,69]$ allows for at least an indirect comparison with existing studies. Further studies, however, should certainly include unimodal conditions to assess the gain of multimodal information in the perception of voice gender.

\section{Conclusions}

We describe dominance of vision over audition in the perception of voice gender behaviourally and neurophysiologically. We observed that top-down influences modulated the processing of bimodal stimuli as early as $40 \mathrm{~ms}$ after stimuli onset, yet this influence depended on the preferential modality for the task, providing 
evidence for a visual bias in the case of face/voice gender categorisation. This bias may be reversed when studying speech perception - a hypothesis to be validated by further studies. Congruency in face and voice stimuli affected neural responses around $190 \mathrm{~ms}$, suggesting that bottom-up multimodal interactions for gender processing are relatively late.

\section{Methods}

\section{Subjects}

Nineteen English-speaking adults (9 women, range = $20-35$ years, mean $=26.4$ years) participated in the study. Subjects reported normal medical history and no hearing problems; all had normal or corrected-to-normal vision. They all provided informed written consent; the experiment was approved by the Sunnybrook Health Sciences Research Ethics Board.

\section{Stimuli and procedure}

Stimuli were bimodal auditory/visual stimulus pairs that were front view greyscale pictures of faces, which subtended a visual angle of $8^{\circ} \times 6^{\circ}$ (see Figure 1, the face stimuli are published with the consent of the models), associated with a voiced word. Previous studies have reported significant findings with the combination of static faces and voices $[27,70]$. Face stimuli were photographs of 3 men and 3 women taken while speaking 14 different words, thus a total of 42 female and 42 male faces. Voice stimuli were 14 monosyllabic French words recorded in stereo from 3 female and 3 male speakers; thus, there were also 42 female and 42 male voice stimuli. The words averaged $300 \mathrm{~ms}$ in duration, including $10 \mathrm{~ms}$ rise and fall times. French words were used with our English-speaking subjects to limit the extent of semantic processing. The voices and faces were randomly associated to form 84 stimuli: 42 were congruent, being female face/female voice and male face/male voice, and 42 were incongruent (i.e., male face/female voice or female face/male voice). Face stimuli were presented for $300 \mathrm{~ms}$ in the centre of a computer screen. Auditory stimuli were normalised for intensity using Matlab; they were presented binaurally through earphones (Etymotic Research, Inc.) at normal speaking levels $(68 \mathrm{~dB} \pm 5 \mathrm{~dB})$. Face stimuli onset was synchronised with the onset of auditory stimuli using Presentation software; interstimulus intervals varied randomly between 1300 and $1600 \mathrm{~ms}$.

The subjects performed three different gender judgment tasks: 1) The first task was to indicate with one of two keys (right and left ctrl key) whether the stimuli were congruent or incongruent in terms of gender, i.e. the subjects had to pay attention to both face and voice gender (BOTH). Subjects completed two blocks of 84 stimuli; response key attribution was counterbalanced across subjects. As this task differed in terms of response mapping it was always run first. 2) Attention was directed towards the faces: subjects performed a gender discrimination of faces (FACE) while ignoring the voices for 84 trials. 3) In the third task they performed gender discrimination of the voices (VOICE) while ignoring the faces for 84 trials. In the latter two tasks, participants pressed one keyboard key (right and left ctrl key) for female and another for male. The order of the presentation of these two tasks was counterbalanced across subjects, as was the response key attribution.

\section{EEG recording and analysis}

The ERPs were recorded in a dimly lit sound-attenuating booth; participants sat $60 \mathrm{~cm}$ from a screen on which stimuli were presented. A fixation cross appeared between presentations and subjects were asked to look at it and refrain from making eye movements. EEG was recorded using an ANT (Advanced Neuro-Technology, Enschede, Netherlands) system and a 64 electrode cap, including three ocular electrodes to monitor vertical and horizontal eye movements. Impedances were kept below $5 \mathrm{k} \Omega$. The sampling acquisition rate was $1024 \mathrm{~Hz}$. FCz was the reference during acquisition; an average reference was calculated off-line.

Continuous EEG was epoched into $600 \mathrm{~ms}$ sweeps including a $100 \mathrm{~ms}$ pre-stimulus baseline. Ocular and muscular artefacts, or trials containing an amplitude shift greater than $100 \mu \mathrm{V}$, were rejected from analyses. Epochs were averaged by condition ( 6 conditions: congruent/incongruent in the 3 tasks) and filtered using a bandpass filter of 1-30 Hz.

Peak analyses were completed on the classical peaks described in the visual, i.e. P1, N170, P2 and VPP (Vertex Positive Potential - [71]), and the auditory ERP literature, i.e. N1, VSR [39]. Unimodal auditory stimuli generally evoke biphasic ERPs, the negative N1, mentioned above, followed by the auditory P2 in fronto-central regions, a positive wave occurring between 160 and $240 \mathrm{~ms}$ after stimulus onset [58]. An auditory P2 was not seen in our data probably due to its temporal coincidence with the VPP, thus being masked by the VPP. Peak latencies and amplitudes were measured for each participant in a $\pm 30 \mathrm{~ms}$ time-window centred on the latencies of the peak in the grand average (visual - P1: $105 \mathrm{~ms}$, N170: $155 \mathrm{~ms}$, VPP: $160 \mathrm{~ms}$ and P2: $220 \mathrm{~ms}$; auditory - N1: $100 \mathrm{~ms}$ and VSR: $350 \mathrm{~ms}$, see Figure 3). $\mathrm{P} 1$ and $\mathrm{P} 2$ were measured at $\mathrm{O} 1 / \mathrm{O} 2, \mathrm{PO} 7 / \mathrm{PO} 8$ and PO3/PO4. N170 was measured at PO9/PO10, PO7/PO8, P7/P8 and P9/P10. VPP was measured at FC1/FC2, FC3/FC4, F1/F2, F3/F4 and C1/C2. Auditory N1 was measured at $\mathrm{FC} 1 / \mathrm{FC} 2, \mathrm{C} 1 / \mathrm{C} 2$ and $\mathrm{CP} 1 / \mathrm{CP} 2$, and $\mathrm{VSR}$ at AF3/AF4, F3/F4 and F1/F2 (see Figure 4a). Latencies 
were measured at one time point per hemisphere at the electrode with the largest amplitude. Amplitudes were taken at this latency at the other selected electrodes over the hemisphere [72].

Peak analyses have been extensively used in ERP literature; however, this technique restrains the analysis to time intervals where a peak is seen. In contrast, spatiotemporal analyses determine when brain activity differs significantly between two conditions and allows ERP differences to be identified independently of peak measures $[1,16]$. Studies of multimodal processing have shown early modulation of brain activity around $40 \mathrm{~ms}[1,73]$ that does not correspond to a precise peak. Thus, we also analysed spatio-temporal effects by comparing brain activity at each time point and electrode.

\section{Statistical analyses}

Behavioural data and peak latencies and amplitudes were submitted to repeated measures analyses of variance (using SPSS11); within subject factors were task (3 levels), stimulus (2 levels) and hemisphere (2 levels) for peak latencies plus electrode (different levels depending on the component) for peak amplitudes. After main effects were assessed, we performed paired comparison and post-hoc tests (for interactions) to determine the factors leading to the effects.

Spatio-temporal effects were assessed by comparing brain activity for the different conditions, at each time point and electrode. Repeated measures ANOVA within the general linear model framework were run on the ERPs using Matlab7.2 with task and stimulus as intersubject factors at each time point and electrode. To estimate the statistical significance of the ANOVA, we calculated a data-driven distribution of F-values using a bootstrap-F method; this method makes no assumption on the normality of the data distribution and is therefore robust to normality violations $[74,75]$. Data were centred at 0 to be under the null hypothesis that conditions do not differ from 0 . ANOVAs at each time point and electrode were run on the centred data after resampling the subjects with replacement. We stored the bootstrapped F-values for each time point and electrode independently. This operation was repeated 999 times to obtain a distribution of 1000 bootstrapped estimates of F-values under the null hypothesis [74]. To correct for multiple comparison, we stored the maximum F-values obtained across all time points in each random sampling loop and for each electrode independently [76]. We then calculated a $95 \%$ confidence interval of the maximum F-values for each electrode. The repeated measures ANOVA was considered significant if the F-value fell outside the bootstrapped 95\% confidence intervals for each time point and electrode (Degrees of freedom (df) are similar for all statistics presented in this study: 2 and 36 for the task factor, 1 and 18 for the stimulus factor and 2 and 36 for the interaction ( $\mathrm{df}$ of factor and error respectively)).

Post-hoc tests were run for the Task factor whenever the ANOVA was significant. Data-driven confidence intervals were calculated for each comparison (VOICE vs. FACE, VOICE vs. BOTH and FACE vs. BOTH). We performed the analyses across subjects by sampling conditions with replacement (electrodes by time points matrices), independently for each subject. For each random sample, we averaged ERPs across subjects independently for each condition, then computed the difference between the averages for the two conditions (for instance VOICE vs. FACE). In each random sampling loop and for each electrode independently, we stored the maximum absolute difference obtained across all time points. This process was repeated 1000 times, leading to a distribution of bootstrapped estimates of the maximum absolute difference between two ERP conditions, averaged across subjects, under the null hypothesis $\mathrm{HO}$ that the two conditions were sampled from populations with similar means. Then the $95 \%$ confidence interval of the mean maximum absolute differences was computed at each electrode (alpha $=0.05$ ). Finally, absolute differences between two sample means at any time point at one electrode were considered significant if they fell outside the H0 95\% confidence interval for that electrode.

\section{Acknowledgements}

Marianne Latinus was supported by la Fondation pour La Recherche Médicale (FRM, FDT20051206128). We thank Dr. Nancy J. Lobaugh for her generosity in allowing us full access to her ERP lab, and the help provided with the studies by Erin Gibson. We would like to thank lan Charest and Guillaume Rousselet for their help in implementing the bootstrap analyses of the ERP data.

\section{Author details}

'Université de Toulouse, UPS, CNRS, Centre de recherche Cerveau et Cognition, Toulouse, France. ${ }^{2}$ Centre for Cognitive Neuroimaging (CCNi) and Department of Psychology, University of Glasgow, Glasgow, G128BQ, UK. ${ }^{3}$ Diagnostic Imaging and Research Institute, Hospital for Sick Children, University of Toronto, Toronto, Ontario, M5G1X8, Canada.

\section{Authors' contributions}

$\mathrm{ML}$ designed the experiment, recorded and analysed the data and wrote the manuscript. RVR helped in analysing the data and writing and reading the manuscript, MJT helped in designing the experiments, writing and reading the manuscript. All authors read and approved the final manuscript.

Received: 27 July 2009 Accepted: 11 March 2010

Published: 11 March 2010

\section{References}

1. Giard $\mathrm{MH}$, Peronnet $\mathrm{F}$ : Auditory-visual integration during multimodal object recognition in humans: a behavioral and electrophysiological study. Journal of Cognitive Neuroscience 1999, 11(5):473-490.

2. Giard MH, Fort A, Mouchetant-Rostaing Y, Pernier J: Neurophysiological mechanisms of auditory selective attention in humans. Front Biosci 2000, 5:D84-94. 
3. Vroomen J, Driver J, de Gelder B: Is cross-modal integration of emotional expressions independent of attentional resources? Cogn Affect Behav Neuroscience 2001, 1(4):382-387.

4. Bertelson P, Radeau M: Cross-modal bias and perceptual fusion with auditory-visual spatial discordance. Percept Psychophys 1981, 29(6):578-584.

5. Driver J: Enhancement of selective listening by illusory mislocation of speech sounds due to lip-reading. Nature 1996, 381(6577):66-68.

6. Spence C, Driver J: Attracting attention to the illusory location of a sound: reflexive crossmodal orienting and ventriloquism. Neuroreport 2000, 11(9):2057-2061.

7. Wada $Y$, Kitagawa N, Noguchi K: Audio-visual integration in temporal perception. Int J Psychophysio/ 2003, 50(1-2):117-124

8. Shimojo S, Shams L: Sensory modalities are not separate modalities: plasticity and interactions. Curr Opin Neurobiol 2001, 11(4):505-509.

9. Bertelson P, Aschersleben G: Temporal ventriloquism: crossmodal interaction on the time dimension. 1. Evidence from auditory-visual temporal order judgment. Int J Psychophysiol 2003, 50(1-2):147-155.

10. Aschersleben $G$, Bertelson P: Temporal ventriloquism: crossmodal interaction on the time dimension. 2. Evidence from sensorimotor synchronization. Int J Psychophysiol 2003, 50(1-2):157-163.

11. de Gelder B, Pourtois $G$, Weiskrantz L: Fear recognition in the voice is modulated by unconsciously recognized facial expressions but not by unconsciously recognized affective pictures. Proc Natl Acad Sci USA 2002, 99(6):4121-4126

12. Fort A, Giard MH: Multi electrophysiological mechanisms of audio-visua integration in human perception. The Handbook of Multisensory Processes Cambridge: MIT PressCalvert GA, Spence C, Stein BE 2004, 503-514.

13. Calvert GA, Brammer MJ, Bullmore ET, Campbell R, Iversen SD, David AS: Response amplification in sensory-specific cortices during crossmodal binding. Neuroreport 1999, 10(12):2619-2623.

14. Eimer M: Crossmodal links in spatial attention between vision, audition, and touch: evidence from event-related brain potentials. Neuropsychologia 2001, 39(12):1292-1303.

15. Calvert GA, Campbell R, Brammer MJ: Evidence from functional magnetic resonance imaging of crossmodal binding in the human heteromodal cortex. Curr Biol 2000, 10(11):649-657.

16. Fort A, Delpuech C, Pernier J, Giard MH: Early auditory-visual interactions in human cortex during nonredundant target identification. Brain Research Cogn Brain Research 2002, 14(1):20-30

17. Teder-Salejarvi WA, MCDonald JJ, Di Russo F, Hillyard SA: An analysis of audio-visual crossmodal integration by means of event-related potential (ERP) recordings. Brain Research Cogn Brain Research 2002, 14(1):106-114.

18. Molholm S, Ritter W, Murray MM, Javitt DC, Schroeder CE, Foxe JJ: Multisensory auditory-visual interactions during early sensory processing in humans: a high-density electrical mapping study. Brain Research Cogn Brain Research 2002, 14(1):115-128.

19. Molholm S, Ritter W, Javitt DC, Foxe JJ: Multisensory visual-auditory object recognition in humans: a high-density electrical mapping study. Cereb Cortex 2004, 14(4):452-465.

20. McGurk H, MacDonald J: Hearing lips and seeing voices. Nature 1976, 264(5588):746-748

21. Besle J, Fort A, Delpuech C, Giard MH: Bimodal speech: early suppressive visual effects in human auditory cortex. Eur J Neuroscience 2004, 20(8):2225-2234

22. Izumi A, Kojima S: Matching vocalizations to vocalizing faces in a chimpanzee (Pan troglodytes). Anim Cogn 2004, 7(3):179-184.

23. Dolan RJ, Morris JS, de Gelder B: Crossmodal binding of fear in voice and face. Proc Natl Acad Sci USA 2001, 98(17):10006-10010.

24. Pourtois G, de Gelder B, Bol A, Crommelinck M: Perception of facial expressions and voices and of their combination in the human brain. Cortex 2005, 41(1):49-59.

25. Pourtois G, de Gelder B, Vroomen J, Rossion B, Crommelinck M: The timecourse of intermodal binding between seeing and hearing affective information. Neuroreport 2000, 11(6):1329-1333.

26. Parr LA: Perceptual biases for multimodal cues in chimpanzee (Pan troglodytes) affect recognition. Anim Cogn 2004, 7(3):171-178.

27. De Gelder B, Vroomen J: The perception of emotions by ear and by eye. Cognition and Emotion 2000, 14(3):289-311.

28. Haxby JV, Hoffman EA, Gobbini MI: The distributed human neural system for face perception. Trends Cogn Sci 2000, 4(6):223-233.
29. Bentin S, Allison T, Puce A, Perez E, McCarthy G: Electrophysiological Studies of Face Perception in Humans. Journal of Cognitive Neuroscience 1996, 8:551-565.

30. George N, Evans J, Fiori N, Davidoff J, Renault B: Brain events related to normal and moderately scrambled faces. Cognitive Brain Research 1996, 4:65-76.

31. Itier RJ, Latinus M, Taylor MJ: Face, eye and object early processing: what is the face specificity? Neuroimage 2006, 29(2):667-676.

32. Rossion B, Gauthier I, Tarr MJ, Despland P, Bruyer R, Linotte S, Crommelinck M: The N170 occipito-temporal component is delayed and enhanced to inverted faces but not to inverted objects: an electrophysiological account of face-specific processes in the human brain. Neuroreport 2000, 11(1):69-74.

33. Puce A, Allison T, McCarthy G: Electrophysiological studies of human face perception. III: Effects of top-down processing on face-specific potentials. Cereb Cortex 1999, 9(5):445-458

34. Severac Cauquil A, Edmonds GE, Taylor MJ: Is the face-sensitive N170 the only ERP not affected by selective attention? Neuroreport 2000, 11(10):2167-2171.

35. Taylor MJ, Edmonds GE, McCarthy G, Allison T: Eyes first! Eye processing develops before face processing in children. Neuroreport 2001, 12(8):1671-1676.

36. Itier RJ, Taylor MJ: Effects of repetition and configural changes on the development of face recognition processes. Dev Sci 2004, 7(4):469-487

37. Bedard C, Belin P: A "voice inversion effect?". Brain Cogn 2004 55(2):247-249

38. Belin P, Fecteau S, Bedard C: Thinking the voice: neural correlates of voice perception. Trends Cogn Sci 2004, 8(3):129-135.

39. Levy DA, Granot R, Bentin S: Processing specificity for human voice stimuli: electrophysiological evidence. Neuroreport 2001, 12(12):2653-2657.

40. Levy DA, Granot R, Bentin S: Neural sensitivity to human voices: ERP evidence of task and attentional influences. Psychophysiology 2003, 40(2):291-305

41. Charest I, Pernet CR, Rousselet GA, Quinones I, Latinus M, Fillion-Bilodeau S, Chartrand JP, Belin P: Electrophysiological evidence for an early processing of human voices. BMC Neurosci 2009, 10:127.

42. Lattner S, Maess B, Wang Y, Schauer M, Alter K, Friederici AD: Dissociation of human and computer voices in the brain: evidence for a preattentive gestalt-like perception. Hum Brain Mapp 2003, 20(1):13-21.

43. Joassin F, Maurage P, Bruyer R, Crommelinck M, Campanella S: When audition alters vision: an event-related potential study of the crossmodal interactions between faces and voices. Neuroscience Lett 2004, 369(2):132-137.

44. Smith EL, Grabowecky M, Suzuki S: Auditory-visual crossmodal integration in perception of face gender. Curr Biol 2007, 17(19):1680-1685.

45. Andersen TS, Tiippana K, Sams M: Factors influencing audiovisual fission and fusion illusions. Brain Research Cogn Brain Research 2004, 21(3):301-308

46. Reddy $L$, Wilken $P$, Koch C: Face-gender discrimination is possible in the near-absence of attention. J Vis 2004, 4(2):106-117.

47. Bindemann $M$, Burton AM, Hooge IT, Jenkins $R$, de Haan $E H$ : Faces retain attention. Psychon Bull Rev 2005, 12(6):1048-1053.

48. Fort A, Delpuech C, Pernier J, Giard MH: Dynamics of cortico-subcortical cross-modal operations involved in audio-visual object detection in humans. Cereb Cortex 2002, 12(10):1031-1039.

49. Degerman A, Rinne T, Pekkola J, Autti T, Jaaskelainen IP, Sams M, Alho K: Human brain activity associated with audiovisual perception and attention. Neuroimage 2007, 34(4):1683-1691.

50. Vuilleumier P: Faces call for attention: evidence from patients with visual extinction. Neuropsychologia 2000, 38(5):693-700

51. Shams L, Kamitani Y, Thompson S, Shimojo S: Sound alters visual evoked potentials in humans. Neuroreport 2001, 12(17):3849-3852.

52. Talsma D, Doty TJ, Woldorff MG: Selective attention and audiovisual integration: is attending to both modalities a prerequisite for early integration? Cereb Cortex 2007, 17(3):679-690.

53. Talsma D, Woldorff MG: Selective attention and multisensory integration: multiple phases of effects on the evoked brain activity. Journal of Cognitive Neuroscience 2005, 17(7):1098-1114.

54. Rossion B, Campanella S, Gomez CM, Delinte A, Debatisse D, Liard L, Dubois S, Bruyer R, Crommelinck M, Guerit JM: Task modulation of brain 
activity related to familiar and unfamiliar face processing: an ERP study. Clin Neurophysiol 1999, 110(3):449-462.

55. Näätänen R, Picton $\mathrm{T}$ : The $\mathrm{N} 1$ wave of the human electric and magnetic response to sound: a review and an analysis of the component structure. Psychophysiology 1987, 24(4):375-425.

56. Alho K, Sams M, Paavilainen P, Naatanen R: Small pitch separation and the selective-attention effect on the ERP. Psychophysiology 1986, 23(2):189-197.

57. Jacques $C$, Rossion $B$ : Concurrent processing reveals competition between visual representations of faces. Neuroreport 2004, 15(15):2417-2421.

58. Michalewski HJ, Prasher DK, Starr A: Latency variability and temporal interrelationships of the auditory event-related potentials (N1, P2, N2, and P3) in normal subjects. Electroencephalogr Clin Neurophysiol 1986, 65(1):59-71.

59. Amedi A, von Kriegstein K, van Atteveldt NM, Beauchamp MS, Naumer MJ: Functional imaging of human crossmodal identification and object recognition. Exp Brain Research 2005, 166(3-4):559-571.

60. Callan DE, Jones JA, Munhall K, Callan AM, Kroos C, Vatikiotis-Bateson E: Neural processes underlying perceptual enhancement by visual speech gestures. Neuroreport 2003, 14(17):2213-2218.

61. Calvert GA, Hansen PC, Iversen SD, Brammer MJ: Detection of audio-visual integration sites in humans by application of electrophysiological criteria to the BOLD effect. Neuroimage 2001, 14(2):427-438.

62. Sestieri C, Di Matteo R, Ferretti A, Del Gratta C, Caulo M, Tartaro A, Olivetti Belardinelli M, Romani GL: "What" versus "where" in the audiovisual domain: an fMRI study. Neuroimage 2006, 33(2):672-680.

63. Talsma D, Kok A, Slagter HA, Cipriani G: Attentional orienting across the sensory modalities. Brain Cogn 2008, 66(1):1-10.

64. Wagner AD: Working memory contributions to human learning and remembering. Neuron 1999, 22(1):19-22.

65. Poldrack RA, Wagner AD, Prull MW, Desmond JE, Glover GH, Gabrieli JD: Functional specialization for semantic and phonological processing in the left inferior prefrontal cortex. Neuroimage 1999, 10(1):15-35.

66. Bushara KO, Grafman J, Hallett M: Neural correlates of auditory-visual stimulus onset asynchrony detection. J Neuroscience 2001, 21(1):300-304.

67. Itier RJ, Taylor MJ: N170 or N1? Spatiotemporal differences between object and face processing using ERPs. Cereb Cortex 2004, 14(2):132-142.

68. Belin P, Zatorre RJ, Lafaille P, Ahad P, Pike B: Voice-selective areas in human auditory cortex. Nature 2000, 403(6767):309-312.

69. Beauchemin M, De Beaumont L, Vannasing P, Turcotte A, Arcand C, Belin P, Lassonde M: Electrophysiological markers of voice familiarity. Eur J Neuroscience 2006, 23(11):3081-3086.

70. Campanella S, Belin P: Integrating face and voice in person perception. Trends Cogn Sci 2007, 11(12):535-543.

71. Jeffreys DA: The influence of stimulus orientation on the vertex positive scalp potential evoked by faces. Experimental Brain Research 1993, 96(1):163-172.

72. Picton TW, Bentin S, Berg P, Donchin E, Hillyard SA, Johnson R Jr, Miller GA, Ritter W, Ruchkin DS, Rugg MD, et al: Guidelines for using human eventrelated potentials to study cognition: recording standards and publication criteria. Psychophysiology 2000, 37(2):127-152.

73. MCDonald JJ, Teder-Salejarvi WA, Hillyard SA: Involuntary orienting to sound improves visual perception. Nature 2000, 407(6806):906-908.

74. Berkovits I, Hancock GR, Nevitt J: Bootstrap resampling approaches for repeated measure designs: relative robustness to sphericity and normality violations. Educational and Psychological Measurement 2000, 60(6):877-892.

75. Wilcox RR: Introduction to Robust Estimation and Hypothesis Testing. Second 2005.

76. Rousselet GA, Husk JS, Pernet CR, Gaspar CM, Bennett PJ, Sekuler AB: Agerelated delay in information accrual for faces: evidence from a parametric, single-trial EEG approach. BMC Neurosci 2009, 10:114.

doi:10.1186/1471-2202-11-36

Cite this article as: Latinus et al:: Top-down and bottom-up modulation in processing bimodal face/voice stimuli. BMC Neuroscience 2010 11:36.

\section{Submit your next manuscript to BioMed Central and take full advantage of:}

- Convenient online submission

- Thorough peer review

- No space constraints or color figure charges

- Immediate publication on acceptance

- Inclusion in PubMed, CAS, Scopus and Google Scholar

- Research which is freely available for redistribution

Submit your manuscript at www.biomedcentral.com/submit
Biomed Central 